\title{
SOLVABLE LINEAR GROUPS OVER DIVISION RINGS ${ }^{1}$
}

\author{
ROBERT L. SNIDER
}

\begin{abstract}
A solvable subgroup of $\mathrm{GL}_{n}(D)$ whose linear span is $M_{n}(D)$ is abelian-by-locally finite.
\end{abstract}

There has recently been a great deal of interest in linear groups over division rings $[3,10-12]$. The theory has mainly been concerned so far with the case in which the division ring is locally finite dimensional over its center. In particular, this case is sufficient to understand what happens when the group is locally finite; there is now a good theory of locally finite linear groups over division rings developed by Zalesskii [12] and Hartley and Shahabi Shojaei [3].

One of the most useful results about linear groups over a field is Malcev's theorem that a solvable irreducible linear group over a field is abelian-by-finite $[\mathbf{6}, \mathrm{p}$. 531]. This is no longer true for linear groups over a division ring. In fact, the solvable subgroups of the multiplicative group $D^{*}$ of the division ring $D$ can be quite complicated. For example, if $G$ is any solvable group which is poly-(torsion-free) abelian, then the group ring has no zero divisors and hence has a division ring of fractions $D$ (see e.g. [8]). It follows that $G$ can be embedded in $D^{*}$. If the zero divisor conjecture is true for group rings of solvable groups, then every torsion-free solvable group can be embedded in some $D^{*}$.

In order to get a positive result, we assume that $G$ is a solvable subgroup of $\mathrm{GL}_{n}(D)$ and that the ring $k\langle G\rangle$ generated by $G$ and the center $k$ of $D$ is simple Artin. In this case, we can prove that $G$ is abelian-by-locally finite. This extra hypothesis is automatically satisfied for irreducible linear groups over a field. We also give an example to show that the locally finite "top" can be arbitrarily bad. Special cases of this theorem when $D$ is locally finite dimensional over its center are due to Zalesskii $[\mathbf{1 2}$, Theorem 6] and Wehrfritz [10, 2.6].

The theorem is interesting from the point of view of group rings. There, one is often interested in homomorphic images of group rings. Here one is really studying the simple Artin images of group rings of solvable groups.

Not only is the theory interesting from the point of view of group rings, the proof uses the theory of group rings, in particular the theory of annihilator free ideals as developed by Zalesskii. The point of view here is that $k\langle G\rangle$ is a homomorphic image of the group ring $k[G]$.

The augmentation ideal of a group ring will be denoted $\omega(G)$. We recall that an ideal $L$ of $k[G]$ is annihilator free if for every infinite subgroup $X$ of $G$, the left annihilator of $\omega(X)+L$ in $k[G] / L$ is zero [6, p. 339]. We will frequently encounter the following situation: $N$ will be a normal subgroup of $G$, and $P$ will be an ideal

Received by the editors August 16, 1983.

1980 Mathematics Subject Classification. Primary 20H25; Secondary 20C07, 16A27.

Key words and phrases. Solvable group, linear group.

${ }^{1}$ This research was partially supported by a grant from the National Science Foundation 
of $k[G]$ such that $P=(P \cap k[N]) k[G]$. In this case the factor ring $k[G] / P$ is a crossed product $k[N] / P \cap k[N] *[G / N]$.

LEMMA. If $G$ is a solvable subgroup of $\mathrm{GL}_{n}(D)$ and $k\langle G\rangle=M_{n}(D)$ where $k$ is the center of $D$, and if for every normal subgroup $N$ of $G$, the ring $k\langle N\rangle$ is a prime ring, then $G$ is abelian-by-locally finite.

Proof. $k\langle G\rangle$ is a homomorphic image of the group ring $k[G]$; hence $k\langle G\rangle=$ $k[G] / P$ for some ideal $P$ of $k[G]$. Let $\boldsymbol{Z}$ denote the Zalesskii subgroup of $G$ (see [6, p. 364]). Suppose $P$ is annihilator free. Then by [6, p. 523], $P=(P \cap k[Z]) k[G]$. Therefore $k[G] / P=k[Z] / P \cap k[Z] *[G / Z]$, a crossed product. Let $t \in G$. Suppose $t^{n} \notin Z$ for all natural numbers $n$. Now $1+t$ is not a zero divisor $\bmod P$. To see this, note that $1+t$ is not a zero divisor in $k[Z] / P \cap k[Z] *\langle t\rangle$ and $k[G] / P$ is a free module over this last ring. Since $1+t$ is not a zero divisor in the simple Artin ring $k[G] / P$, it must be invertible. Again this is impossible since $1+t$ is clearly not invertible in $k[Z] / P \cap k[Z] *\langle t\rangle$ and $k[G] / P$ is a free module over $k[Z] / P \cap k[Z] *\langle t\rangle$. Therefore $G / Z$ is periodic. $Z$ is an FC-group whence $Z$ is center-by-locally finite $[7$, p. 121]. Therefore $G$ is abelian-by-locally finite.

It suffices then to prove that $P$ is annihilator free. In order to prove this, it suffices to show $P \cap k[Z]$ is an annihilator free ideal of $k[Z][6$, p. 523]. Suppose it is not annihilator free. Let $X$ be an infinite subgroup of $Z$ such that the left annihilator of $\omega(X)+(P \cap k[Z])$ is nonzero. Since $Z$ is an FC-group, it is center-bylocally finite. Let $A=Z(Z)$. Since $A$ is normal in $G, k\langle A\rangle$ is an integral domain. The nonzero elements $S$ of $k\langle A\rangle$ form an Ore set for $k\langle A\rangle$. Since $A$ is normal in $G, S$ is a right divisor set for $k\langle G\rangle$ (see the proof of Lemma 3.5, p. 609 of [6]). Since $k[G] / P$ is simple Artin, $S$ consists of nonzero divisors [6, p. 494]. If $x \in X$, there exists an integer $n$ with $x^{n} \in A$ whence $1-x^{n} \in k\langle A\rangle$. But $1-x^{n}$ is a zero divisor, hence $1-x^{n}=0$ and $x^{n}=1$. It follows that $X \subset T$ where $T$ is the torsion subgroup of the FC-group $Z$. Since $T$ is normal in $G, k\langle T\rangle$ is prime. $T$ is locally finite whence $k\langle T\rangle$ is an algebraic algebra. It follows that the Jacobson radical of $k\langle T\rangle$ is nil [6, p. 53] and hence nilpotent [4, p. 71]. Therefore the Jacobson radical is zero and hence by Proposition 1 of $[\mathbf{1 2}], k\langle T\rangle$ is Artin and hence simple Artin. It follows from [12] that $T$ is metabelian-by-finite. Let $M$ be a normal metabelian subgroup of $T$ of finite index. Let $F$ be the Hirsch-Plotkin radical of $T$. As was argued above, $k\langle F\rangle$ is simple Artin. In char $p>0$, the proof of Theorem 1 of [12] implies $F$ is actually a linear group over a field. Since $F$ is locally nilpotent, in characteristic 0 , Proposition 3 of $[\mathbf{1 2}]$ also implies that $F$ is linear over a field. As an FC-linear group, $F$ is center-by-finite $[9$, p. 16]. Let $B=Z(F)$. Since $X$ is an infinite locally finite group, it has an infinite abelian subgroup [7, p. 95]. We may replace $X$ with this subgroup and assume $X$ is abelian. Also we may replace $X$ with $X \cap M$ and assume $X \subset M$. Let $D=M^{\prime} . \quad D \subset F$. Hence we have $[D: B \cap D]<\infty$. Since $B$ is normal in $G, k\langle B\rangle$ is an integral domain; therefore, as above, the nonzero elements of $k\langle B\rangle$ are not zero divisors. In particular, $1-b$ is not a zero divisor for each $b \in B$. It follows that $X \cap B=1$. Two cases arise.

Case 1. $\left|C_{X}(B)\right|=\infty$. In this case, replace $X$ with $C_{X}(B)$. Now $X D$ has $X(B \cap D)$ as an abelian subgroup of finite index. $X D$ contains a characteristic abelian subgroup $N$ of finite index $[6$, p. 530]. $X D$ and hence $N$ are normal in $M$, hence

$$
N \subset \operatorname{Fitt}(M) \subset F .
$$


$[N: B \cap N]<\infty$ whence $[X: X \cap B]<\infty$. But $X \cap B=1$ and hence $X$ is finite, a contradiction.

Case 2. $\left|C_{X}(B)\right|<\infty$. Suppose $X$ has a finite subgroup $Y$ with $Y \cap C(B)=1$ and $|Y|>n$. Note $k\langle B\rangle$ is an integral domain since $B$ is normal in $G$. Also $k\langle B\rangle$ is an algebraic extension of $k$ and hence $k\langle B\rangle$ is a field. Since $Y$ acts faithfully on $k\langle B\rangle$, the ring $k\langle B Y\rangle$ is isomorphic to a classical crossed product $k\langle B\rangle * Y$. Furthermore, the factor set is trivial since $B \cap Y=1$. Therefore $k\langle B Y\rangle$ is an $m \times m$ matrix ring where $m=|Y|$. But this is impossible since $k\langle B Y\rangle \subset M_{n}(D)$ and hence there are at most $n$ orthogonal idempotents in $k\langle B Y\rangle$. Therefore every subgroup of $X$ with at least $n+1$ elements intersects the finite group $C_{x}(B)$. Therefore $X$ is a finite rank abelian group. Also $X$ is a $\pi$-group for some finite set $\pi$ of primes. $B X$ is an FC-group whence $B X / Z(B X)$ is residually finite [7, p. 121]. Clearly $Z(B X) \cap X$ is finite. But $X / Z(B X) \cap X$ is a finite rank periodic abelian group and residually finite. Therefore $X$ has no $p^{\infty}$-sections and hence $X$ is finite, a contradiction.

THEOREM. If $G$ is a solvable subgroup of $\mathrm{GL}_{n}(D)$ and $k\langle G\rangle=M_{n}(D)$ where $k$ is the center of $D$, then $G$ is abelian-by-locally finite.

PROOF. We will induct on $n$. By the Lemma, we may assume that there exists a normal subgroup $N$ of $G$ such that $k\langle N\rangle$ is not prime. Let $R$ be the prime radical of $k\langle N\rangle$. Since $R$ is a nil subring of $M_{n}(D), R$ is nilpotent [4, p. 71] and $G$-invariant. Therefore $R k\langle G\rangle$ is a nilpotent ideal of $k\langle G\rangle$, and hence $R$ is zero. Since $k\langle N\rangle$ has ACC on annihilators, $k\langle N\rangle$ has only a finite number of minimal prime ideals and each of these is an annihilator ideal [1, p. 13]. Therefore each minimal prime has finite orbit under conjugation by $G$. Hence there exists a normal subgroup $H$ of finite index with $N \subset H$ such that $H$ stabilizes each minimal prime of $k\langle N\rangle$. The ring $k\langle G\rangle$ is a finite normalizing extension of $k\langle H\rangle$. It follows that $k\langle H\rangle$ is Artin [2]. As above its prime radical is zero whence $k\langle H\rangle$ is semisimple Artin. Therefore $k\langle H\rangle=\bigoplus_{r=1}^{t} S_{i}$ where the $S_{i}$ are simple Artin rings. Let $P$ be a minimal prime ideal of $k\langle N\rangle . P$ is an annihilator ideal whence $P=\operatorname{Rt} . A n n_{k\langle N\rangle}(I)$ for some $I$. But $P k\langle H\rangle$ is a two-sided ideal of $k\langle H\rangle$. Also Lt.Ann $k\langle N\rangle(P k\langle H\rangle) \neq 0$ whence $P k\langle H\rangle$ is a proper ideal and $t>1$. Let $\pi_{i}$ be the projection of $k\langle H\rangle$ onto $S_{i}$. By induction, $\pi_{i}(H)$ is abelian-by-locally finite. $H$ is isomorphic to a subgroup of $\pi_{1}(H) \times \cdots \times \pi_{t}(H)$ and hence $H$ is abelian-by-locally finite. Let $A$ be a normal abelian subgroup of $H$ with $H / A$ locally finite. Let $A_{1}, \ldots, A_{m}$ be the $G$-conjugates of $A$. Note $A_{1} \cap \cdots \cap A_{i} / A_{1} \cap \cdots \cap A_{i} \cap A_{i+1}$ is isomorphic to a subgroup of $H / A_{i+1}$ and hence is locally finite. It follows that $G / A_{1} \cap \cdots \cap A_{m}$ is locally finite.

We now give an example to show the locally finite "top" in the Theorem can be arbitrary. Let $L$ be a locally finite group. $L=F / R$ for some free group $F$. Let $H=F / R^{\prime}$. $H$ is torsion free and the group ring $k[H]$ has a division ring of fractions $D[\mathbf{5}]$. Let $B=R / R^{\prime}$. Let $S$ be the nonzero elements of $k[B] . S$ is an Ore set for $k[B]$ and hence an Ore set for $k\left[H_{1}\right]$ for any $B \subset H_{1} \subset H[6$, p. 609]. If $H_{1} \supset B$ with $H_{1} / B$ finite, $S^{-1} k\left[H_{1}\right]$ has no zero divisors (as a subring of $D$ ) and is finite dimensional over the field $S^{-1} k[B]$. Hence $S^{-1} k\left[H_{1}\right]$ and hence $S^{-1} k[H]$ is a division ring. Let $A$ be the nonzero elements of $S^{-1} k[B]$. Let $G=A H$. Then $k\langle G\rangle=S^{-1} k\langle H\rangle=D$ and $G / A \simeq L$. 


\section{REFERENCES}

1. A. W. Chatters and C. R. Hajarnavis, Rings with chain conditions, Pitman, London, 1980.

2. E. Formanek and A. V. Jategaonkar, Subrings of Noetherian rings, Proc. Amer. Math. Soc. 46 (1974), 181-186.

3. B. Hartley and M. A. Shahabi Shojaei, Finite groups of matrices over division rings, Math. Proc. Cambridge Philos. Soc. 92 (1981), 55-64.

4. N. Jacobson, The theory of rings, Math. Surveys, no. 2, Amer. Math. Soc., Providence, R.I., 1943.

5. A. I. Lichtman, On embedding of group rings in division rings, Israel J. Math. 23 (1976), 288-297.

6. D. S. Passman, The algebraic structure of group rings, Wiley, New York, 1977.

7. D. J. S. Robinson, Finiteness conditions and generalized soluble groups, Vol. 1, Springer-Verlag, New York, 1972.

8. R. L. Snider, The zero divisor conjecture for some solvable groups, Pacific J. Math. 90 (1980), 191-196.

9. B. A. F. Wehrfritz, Infinite linear groups, Springer-Verlag, New York, 1973.

10. __ Nilpotence in certain linear groups over division rings, Proc. London Math. Soc. (3) 46 (1983), 334-346.

11. _ The upper central factors of certain skew-linear groups, Arch. Math. (Basel) 40 (1983), 481-494.

12. A. E. Zalesskii, The structure of several classes of matrix groups over a division ring, Siberian Math. J. 8 (1967), 978-988.

Department of Mathematics, Virginia Polytechnic Institute \& State UniVERSITY, BLACKSBURG, VIRGINIA 24061-4097 